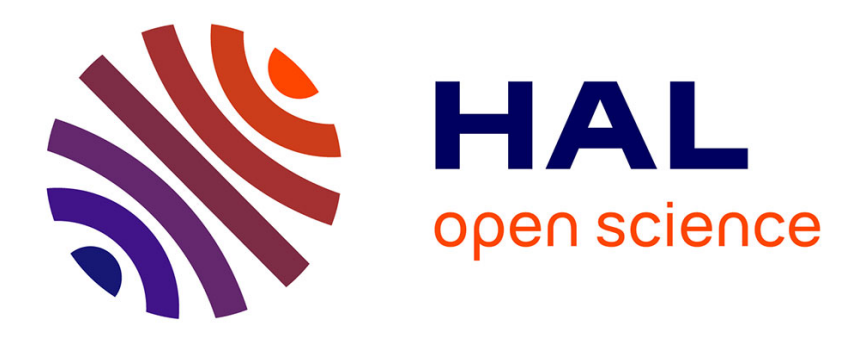

\title{
Sur une classification topologique des courbes de distribution de masse des fragments de fission
}

R. da Silveira

\section{To cite this version:}

R. da Silveira. Sur une classification topologique des courbes de distribution de masse des fragments de fission. Journal de Physique Lettres, 1979, 40 (14), pp.319-322. 10.1051/jphyslet:019790040014031900 . jpa-00231634

\section{HAL Id: jpa-00231634 https://hal.science/jpa-00231634}

Submitted on 1 Jan 1979

HAL is a multi-disciplinary open access archive for the deposit and dissemination of scientific research documents, whether they are published or not. The documents may come from teaching and research institutions in France or abroad, or from public or private research centers.
L'archive ouverte pluridisciplinaire HAL, est destinée au dépôt et à la diffusion de documents scientifiques de niveau recherche, publiés ou non, émanant des établissements d'enseignement et de recherche français ou étrangers, des laboratoires publics ou privés. 


\title{
LE JOURNAL DE PHYSIQUE-LETTRES
}

\section{Sur une classification topologique des courbes de distribution de masse des fragments de fission}

\author{
R. da Silveira \\ Division de Physique Théorique $\left({ }^{*}\right)$, Institut de Physique Nucléaire, 91406 Orsay, France
}

(Reçu le 2 avril 1979, accepté le 23 mai 1979)

\begin{abstract}
Résumé. - Nous proposons une classification des courbes de distribution de masse des fragments de fission, d'après leur morphologie, en utilisant un modèle topologique de la Théorie des Catastrophes. Cette classification laisse à supposer que la composante asymétrique, bien connue pour la fission des noyaux au-delà du Fr, pourrait émerger et s'accentuerait graduellement à partir du $\mathrm{Bi}$ ou du $\mathrm{Po}$.
\end{abstract}

\begin{abstract}
Using a topological model of Catastrophe Theory, we propose a classification of the yield-mass curves of fission fragments according to their morphologies. From this classification it appears that the well known asymmetric component for fission of nuclei heavier than $\mathrm{Fr}$, would probably emerge and gradually increase from $\mathrm{Bi}$ or Po.
\end{abstract}

La rupture des noyaux en deux fragments de masses à peu près égales (fission symétrique) ou très différentes (fission asymétrique) est peut-être l'aspect le plus intrigant du processus de fission et reste de nos jours, malgré des progrès considérables, un point passablement obscur $[1,2,4]$.

Les données expérimentales de distribution de masse des fragments de fission (voir la figure 3) se présentent sous la forme de courbes, pouvant exhiber un, deux ou trois maxima. Suivant l'élément considéré d'une part et l'énergie d'excitation d'autre part, on peut obtenir respectivement une des trois formes citées ou encore, une transition continue entre deux d'entre elles. Cette systématique qualitative suggère naturellement d'essayer d'organiser entre elles les courbes expérimentales de manière à constituer un tableau dont on pourrait dégager certaines propriétés globales. Quelques démarches empiriques dans ce sens ont été esquissées et sont connues depuis longtemps dans la littérature $[3,4]$.

Cela étant, l'objet de ce travail est de montrer que l'on peut, à l'aide des modèles topologiques de la Théorie des Catastrophes [5], se donner les critères rigoureux permettant d'assembler les courbes expérimentales d'après leur morphologie dans une sorte de puzzle, dont la cohérence interne permettrait un éclairage nouveau de la situation.
Soit donc $P(\eta), \eta=\left(M_{1}-M_{2}\right) / M$, la probabilité de fission d'un noyau de masse $M$ en deux fragments de masses $M_{1}$ et $M_{2}$. La fonction $P(\eta)$ dépend, outre de la variable $\eta$ supposée continue, d'un certain nombre de paramètres $\{C\}$. Mais la forme explicite de la fonction $P(\eta ; C)$, ne nous intéresse pas car notre objectif, rappelons-le, est de faire une classification morphologique des courbes de distribution de masse et nullement d'en faire une étude quantitative. Par contre, nous devons dénombrer parmi les paramètres $\{C\}$ ceux dont la variation révèle expérimentalement les différentes formes de ces courbes. Pour observer ces formes nous avons à notre disposition trois paramètres : l'énergie d'excitation $E$, le nombre de protons $Z$ et le nombre de neutrons $N$. Mais l'expérience montre qu'il suffit de considérer les deux premiers $(E, Z)$, puisque l'on observe pour différents isotopes du même élément $[8,9,10,15,18]$ des courbes de distribution de masse morphologiquement analogues. Cela dit, pour faire la classification morphologique dont il est question, il faut maintenant connaître toutes les manières dont les deux paramètres $Z$ et $E$ peuvent faire émerger ou disparaître les maxima de $P(\eta ; Z, E)$, ce qui revient à déterminer les valeurs $(Z, E)$ pour lesquelles cette fonction admet des points critiques dégénérés, lesquels sont définis par

$$
\frac{\mathrm{d}}{\mathrm{d} \eta} P(\eta ; Z, E)=0, \frac{\mathrm{d}^{2}}{\mathrm{~d} \eta^{2}} P(\eta ; Z, E)=0 .
$$

(*) Laboratoire associé au C.N.R.S. 
Le problème ainsi posé a une réponse simple dans le cadre de la Théorie des Catastrophes [5]. Le lieu géométrique défini par les valeurs $(Z, E)$ obtenues en éliminant $\eta$ entre les deux éqs. (1) constitue l'ensemble catastrophe de bifurcation [5] de la fonction $P(\eta ; Z, E)$. Or cette fonction ne dépend (morphologiquement) que de deux paramètres et est, pour des raisons physiques évidentes, une fonction paire de $\eta$. Le théorème de classification de Thom [5] nous apprend alors qu'il existe un difféomorphisme (i.e. un changement d'axes qui conserve topologicodifférentiellement les formes) qui échange localement l'ensemble catastrophe de $P(\eta ; Z, E)$ avec celui de la forme canonique (le papillon dual suivant la nomenclature consacrée)

$$
V(x)=-\left(\frac{x^{6}}{6}+a \frac{x^{4}}{4}+b \frac{x^{3}}{3}+c \frac{x^{2}}{2}+d x\right)
$$

avec

$$
b=d=0 \text {. }
$$

Comme seules les morphologies engendrées par $(Z, E)$ dans $P(\eta)$ nous intéressent, nous sommes de la sorte assurés de les retrouver dans $V(x)$ lorsqu'on se déplacera dans l'espace des paramètres $[a, c]$. Reste, bien entendu, à postuler que l'ensemble catastrophe de $P(\eta)$ est non seulement localement mais aussi globalement (i.e. dans le domaine de $(Z, E)$ qui nous intéresse) celui de la forme canonique (2). L'ensemble catastrophe de $V(x)$ dans le plan $[c, a]$ avec $b=d=0$ est dessiné dans la figure 1 .

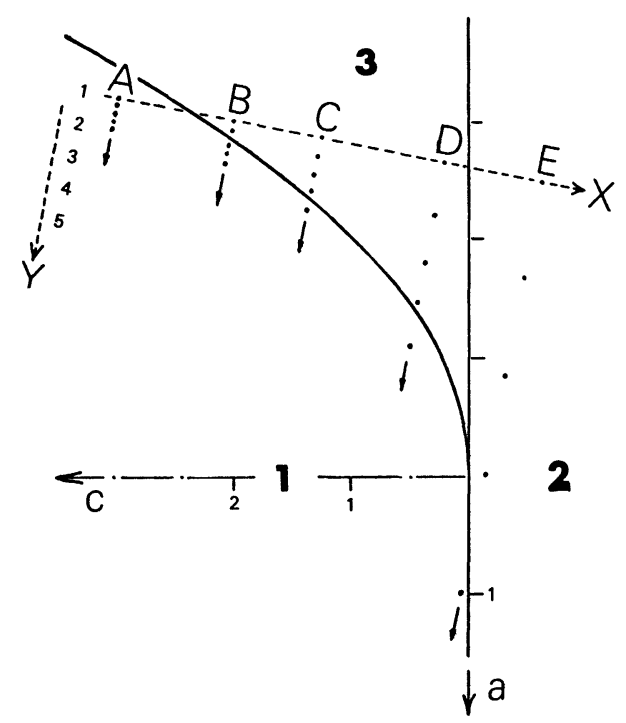

Fig. 1. - Les lignes continues représentent l'ensemble catastrophe de bifurcation de la fonction $V(x)$ dans le plan $[a, c]$, pour $b=d=0$. Les points $\mathrm{A}(I), \mathrm{B}(I), \mathrm{C}(I), \mathrm{D}(I), \mathrm{E}(I), I=(1, \ldots, 5)$, sont disposés de manière à reproduire qualitativement les formes des courbes de distribution de masse (voir le texte).

[Full lines : the catastrophe set of the function $V(x)$ in the plane $[a, c]$ for $b=d=0$. The distribution of the points $\mathrm{A}(I), \mathrm{B}(I)$, $\mathrm{C}(I), \mathrm{D}(I), \mathrm{E}(I), I=(1, \ldots, 5)$ is such that the qualitative forms of the mass-yield curves are reproduced (see text).]
Il est constitué de la droite $c=0$ et de la branche de parabole $a^{2}=4 c, a \leqslant 0$. Ces deux lignes délimitent trois zones $(1,2,3)$ où $V(x) \mathrm{a}$, respectivement, un, deux et trois maxima.

Passons maintenant à l'exploitation de notre modèle. Pour ce faire, procédons par étapes et considérons d'abord les formes typiques des courbes de distribution de masse. Pour des énergies d'excitation n'excédant l'énergie du seuil que d'une dizaine de $\mathrm{MeV}$ environ, on constate [1, 2, 4] que : 1) Pour les éléments faiblement fissiles $(Z \lesssim 81)$ la fission est symétrique. 2) Pour les éléments très lourds $(Z>91)$ la fission est asymétrique. 3) Dans une région intermédiaire $(Z \sim 88,89)$, on observe les deux modes de fission avec une importance comparable. Enfin, lorsque l'énergie d'excitation augmente, toutes les distributions de masse deviennent peu à peu symétriques. Passons maintenant à l'espace $[c, a]$, figure 1, et considérons la droite $X$. Sur celle-ci et dans chacune des trois zones qu'elle traverse, choisissons un point en position générale, i.e. pas trop voisin des lignes de transition de forme (les points se trouvant sur ces lignes déterminent des formes structurellement instables [5]). Soient les points $A(1), C(1)$ et $E(1)$. Pour chacun de ces trois points on trouve dans la première rangée de la figure 2 les formes de $V(x ; a, c)$ correspondantes. Celles-ci reproduisent morphologiquement les courbes expérimentales typiques à basse énergie. Maintenant, partant de chacun de ces trois points dans une direction $Y$ perpendiculaire à $X$, considérons successivement une série de points (cinq avec le point de départ) disposés de manière à couvrir, dans chaque cas, les zones morphologiquement différentes. On trouve alors les séquences de formes $\mathrm{A}(1,2,3,4,5), \mathrm{C}(1,2,3,4,5)$ et $\mathrm{E}(1,2,3,4,5)$, figure 2 , qui reproduisent l'évolution des distributions de masse lorsque l'énergie augmente. L'évolution continue des formes, qui se dégage de notre modèle, laisse à penser que les distributions de masse de certains éléments pourraient exhiber des formes faisant la transition entre deux formes typiques successives. Ces formes de transition sont d'autant plus intéressantes à dégager qu'elles sont souvent plus difficiles à reconnaître ou à préciser expérimentalement. Considérons alors les points $\mathrm{B}(1)$ et $\mathrm{D}(1)$, figure 1 , voisins des frontières séparant deux domaines. En procédant comme précédemment, considérons les séquences de figures $\mathrm{B}(1,2,3,4,5)$ et $\mathrm{D}(1,2,3,4,5)$, figure 2 , que l'on obtient en partant de ces deux points.

La figure $B(1)$ illustre la transition entre la fission purement symétrique $(\mathrm{A}(1))$ et celle $(\mathrm{C}(1))$ où symétrie et asymétrie sont d'importance comparable. La figure $\mathrm{D}(1)$, transition entre $\mathrm{C}(1)$ et $\mathrm{E}(1)$, illustre les situations où la symétrie, absente près du seuil, s'installe peu à peu lorsque l'énergie augmente. Ainsi, avec à la fois les formes typiques et celles de transition, le tableau de la figure 2 est à présent complet. En suivant les mêmes critères qui ont permis 


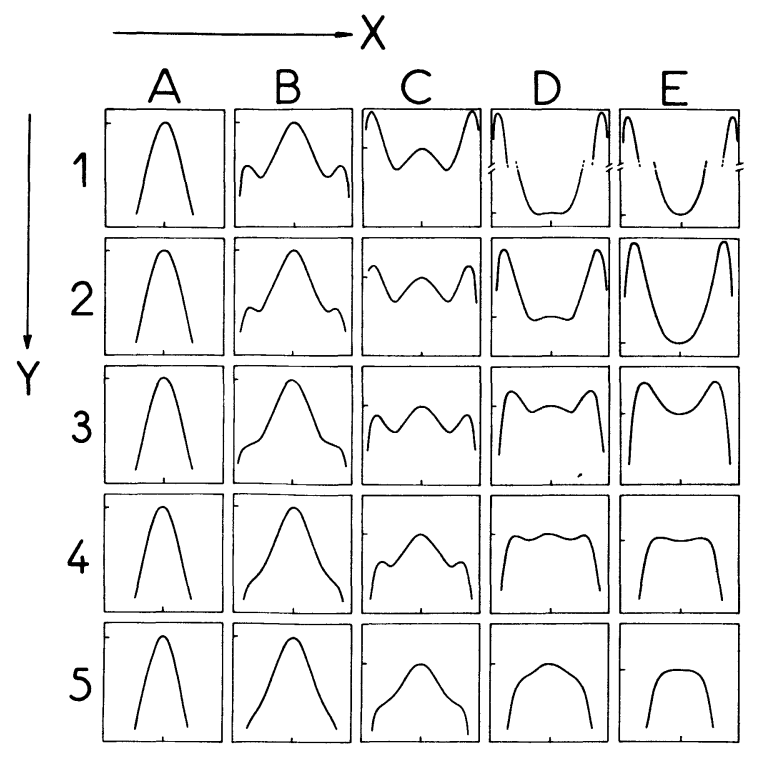

Fig. 2. - Représentation graphique de la fonction

$$
V=-\left[x^{6} / 6+a x^{4} / 4+c x^{2} / 2\right] .
$$

Pour chaque case, les valeurs de $a$ et de $c$ sont celles des coordonnées de chaque point $\mathrm{A}(I), \mathrm{B}(I), \mathrm{C}(I), \mathrm{D}(I), \mathrm{E}(I), I=(1, \ldots, 5)$ de la figure 1. Les échelles, ordonnées et abscisses, sont linéaires (le zéro est repéré par un trait). Pour conserver la même échelle partout, nous avons dû tronquer les dessins des figures $\mathrm{D}(1)$ et $\mathrm{E}(1)$.

[The graphical representation of the function

$$
V=-\left[x^{6} / 6+a x^{4} / 4+c x^{2} / 2\right] .
$$

For each figure the values of $a$ and $c$ are those of the coordinates of each point $\mathrm{A}(I), \mathrm{B}(I), \mathrm{C}(I), \mathrm{D}(I), \mathrm{E}(I), I=(1, \ldots, 5)$, as in figure 1 . The scales of the ordinates and abscissae are linear (the zero being indicated by the marks in each case). To keep the same scale throughout we have cut figures $\mathrm{D}(1)$ and $\mathrm{E}(1)$.]

de l'établir, essayons maintenant d'en construire un, avec les données expérimentales à notre disposition. L'utilisation de ces données appelle quelques remarques (voir la réf. [1] pour un aperçu plus détaillé). Pour la fission induite $\left(\mathrm{p}, \mathrm{d},{ }^{3} \mathrm{He}, \alpha, \ldots\right)$ et pour autant que l'énergie du projectile reste modérée ( $\lesssim 40 \mathrm{MeV}$ ) on peut négliger l'éjection de particules chargées $(p, d, \alpha)$ par interaction directe et supposer que la charge du noyau fissionnant est celle du noyau composé. Par contre le nombre de masse de ce dernier n'est connu, dans bon nombre de cas, qu'à quelques unités près en raison de la compétition évaporationfission. Par conséquent nous supposerons que les noyaux composé et fissionnant sont de la même espèce, sans chercher à préciser de quel isotope il s'agit. Cela dit, considérons d'abord les formes typiques, que nous repérerons sur la figure 3 comme nous l'avons fait pour la figure 2. Commençons par les noyaux très lourds, domaine le plus riche en données expérimentales (voir les réfs. [1, 2, 4] ainsi que celles qui y sont données). Pour l'U et au-delà, on constate que les distributions de masse évoluent à partir d'une asymétrie très marquée (fission spon-

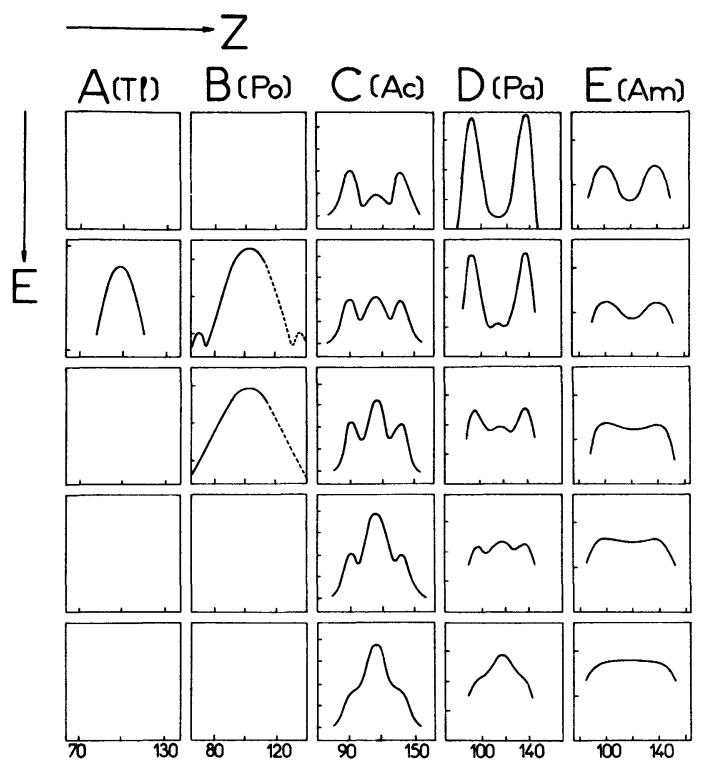

Fig. 3. - Courbes expérimentales de distribution de masse des fragments de fission obtenues pour les réactions suivantes (colonne par colonne et de haut en bas) : A) ${ }^{197} \mathrm{Au}+\alpha, 42 \mathrm{MeV}$ [11]. B) ${ }^{209} \mathrm{Bi}+\mathrm{p}, 36,56 \mathrm{MeV}[13]$. C) ${ }^{226} \mathrm{Ra}\left({ }^{3} \mathrm{He}\right.$, d) ${ }^{227} \mathrm{Ac}, 23,5 \mathrm{MeV}$ [10]; ${ }^{226} \mathrm{Ra}+\mathrm{p}, 11[8], 12,5,15,5 \mathrm{MeV}$ [9]; ${ }^{226} \mathrm{Ra}+\mathrm{d}$, $19 \mathrm{MeV}$ [9]. D) ${ }^{232} \mathrm{Th}+\mathrm{p}, 13,20,35,45,53 \mathrm{MeV}$ [16]. E) ${ }^{239} \mathrm{Pu}+\mathrm{d}$, $9,2,12,3,16,1,17,9,23,4 \mathrm{MeV}$ [7]. Echelles des figures : celle des ordonnées (proportionnelle au nombre de coups) est linéaire pour les figures de la colonne $C$ (le premier trait repère le zéro) et logarithmique pour les autres (chaque trait est un ordre de grandeur). Les abscisses sont en unités de masse.

[Experimental data of mass-yield curves of fission fragments obtained from the following reactions (column by column and from top to bottom) : A) ${ }^{197} \mathrm{Au}+\alpha, 42 \mathrm{MeV}$ [11]. B) ${ }^{209} \mathrm{Bi}+\mathrm{p}$, 36, $56 \mathrm{MeV}[13] . \mathrm{C}){ }^{226} \mathrm{Ra}\left({ }^{3} \mathrm{He}, \mathrm{d}\right){ }^{227} \mathrm{Ac}, 23.5 \mathrm{MeV}[10] ;{ }^{226} \mathrm{Ra}+\mathrm{p}$, 11 [8], 12.5, 15.5 MeV [9]; ${ }^{226} \mathrm{Ra}+\mathrm{d}, 19 \mathrm{MeV}$ [9]. D) ${ }^{232} \mathrm{Th}+\mathrm{p}$, 13, 20, 35, 45, $53 \mathrm{MeV}[16]$. E) ${ }^{239} \mathrm{Pu}+\mathrm{d}, 9.2,12.3,16.1,17.9$, $23.4 \mathrm{MeV}$ [7]. Scales of the figures : ordinates (proportional to the number of counts) are linear for the figures of column $\mathrm{C}$ (the first mark indicating the zero) and are logarithmic for all the other columns. Also for the other columns, each mark is an order of magnitude greater. The abscissae are in mass units.]

tanée ou voisine du seuil) vers la symétrie. Nous remplissons la colonne $\mathrm{E}$ (l'énergie augmentant de haut en bas), avec les données de la réaction ${ }^{239} \mathrm{Pu}+\mathrm{d} \rightarrow \mathrm{Am} \quad(9,2 ; 12,3 ; 16,1 ; 17,9$; $23,4 \mathrm{MeV}$ ) [7]. Pour la région intermédiaire, colonne C, nous utiliserons les données de trois réactions différentes. Pour la première, ${ }^{226} \mathrm{Ra}\left({ }^{3} \mathrm{He}, \mathrm{d}\right){ }^{227} \mathrm{Ac}$ $(23,5 \mathrm{MeV})$ [10] il a été établi que les fragments de fission provenaient de noyaux $d^{\mathbf{2 2 7}} \mathrm{Ac}$ ayant entre 7 et $13 \mathrm{MeV}$ d'énergie d'excitation. Les deux autres réactions sont ${ }^{226} \mathrm{Ra}+\mathrm{p} \rightarrow$ Ac $(11$ [8]; 12,5; 15,5 [9] MeV) et ${ }^{226} \mathrm{Ra}+\mathrm{d} \rightarrow \mathrm{Ac}(19 \mathrm{MeV})$ [9]. A cette zone intermédiaire appartient aussi le $\mathbf{R a}$ dont la distribution de masse pour la fission induite par des $\gamma$ de $23 \mathrm{MeV}$ [12] est très voisine de celle obtenue par ${ }^{226} \mathrm{Ra}+\mathrm{p}(11 \mathrm{MeV}$ [8]). Plus près du seuil il n'y a pas de mesures de distribution de masse mais il a été établi $[10,20]$ que, comme pour l'Ac, l'asymétrie s'accentue. La zone A concerne les noyaux 
faiblement fissiles et est assez pauvre en données expérimentales. Dans la colonne correspondante figurent les données de la réaction ${ }^{197} \mathrm{Au}+\alpha \rightarrow \mathrm{Tl}$, (42 MeV) [11]. En l'absence de données pour des énergies plus proches du seuil, nous laissons vide la première case. Passons à la zone de transition $B$. Cette zone est expérimentalement encore très floue car, pour y déceler l'émergence de l'asymétrie, il faut des données précises à des taux de comptage très faibles. Sur la colonne $B$ figurent les données de la réaction ${ }^{209} \mathrm{Bi}+\mathrm{p} \rightarrow \mathrm{Po}(36,56 \mathrm{MeV})[13]$. En remplissant cette colonne nous avons, comme précédemment, laissé la première case vide. On peut penser en effet que plus près du seuil l'importance relative de l'asymétrie devrait augmenter, comme le suggère la colonne B de la figure 2. Cette zone de transition, où l'asymétrie s'installe peu à peu, devrait s'étendre sur plusieurs unités de $Z$ allant vraisemblablement du Bi au Fr. Pour le Rn et le Fr il n'existe pas (à ma connaissance) de données de distribution de masse. Pour le Bi [12] et l'At [14, 15, 17, 19], ces données ont été obtenues à énergie trop élevée ou ne sont pas assez précises pour permettre d'infirmer ou de confirmer cette prévision. Reste la zone de transition D où la symétrie n'apparaît qu'au-delà du seuil. Cette zone est facilement identifiable sur différentes données où le $\mathrm{Th}[15,17]$ et le $\mathrm{Pa}[15,16]$ sont formés en tant que noyau composé. Sur la colonne D, figurent les résultats de la réaction ${ }^{232} \mathrm{Th}+\mathrm{p} \rightarrow \mathrm{Pa}(13,20,35$, 45, $53 \mathrm{MeV}$ ) [16].

Essayons de tirer, très succinctement, quelques conclusions. Lorsqu'on examine la figure 3 à la lumière du modèle topologique de la figure 2 on dégage une évolution apparemment continue et cohérente des composantes symétrique et asymétrique de la fission. Cette évolution est nettement perçue lorsqu'on va du $\mathrm{Ra}$ vers l'U mais elle reste à préciser dans la zone qui s'étend du Bi au Fr. Les données de la colonne B, quoique isolées, semblent indiquer qu'une telle évolution pourrait bien exister.

Remerciements. - Je remercie vivement MM. M. Demeur et $P$. Pirès avec lesquels j'ai eu de très fructueuses discussions.

\section{Bibliographie}

[1] Lefort, M., La Chimie Nucléaire, Chap. VII (Dunod) 1966.

[2] Vandenbosch, R. and Huizenga, J. R., Nuclear Fission, Chap. XI (Academic Press) 1973.

[3] Fairhall, A. W., in Theories of Nuclear Fission de L. Willets (Clarendon Press) 1964.

[4] HydE, E. K., The Nuclear Properties of the Heavy Elements, vol. III (Prentice-Hall) 1964.

[5] Тном, R., Stabilité Structurelle et Morphogenèse (Interéditions) 1972.

Tном, R., Modèles Mathématiques de la Morphogenèse (Editions 10-18) 1974. (Pour un abord plus élémentaire du sujet, voir la référence [6].)

[6] Zeeman, E. C., Catastrophe Theory (Addison-Wesley) 1977.

Poston, T. and StewarT, I., Catastrophe Theory and its Applications (Pitman) 1978.

DA SilveIRA, R., Théorie des Catastrophes et Physique Nucléaire, Rapport Orsay, IPNO/TH 78-34.

[7] Gibson, W. M., Rapport UCRL-3493 (1956).

[8] SChmith, H. W., DabBs, J. W. T. and MilleR, P. D., Symposium on the Physics and Chemistry of Fission, Salzburg, vol. I (1965) 517.

[9] Perry, D. G. and Fairhall, A. W., Phys. Rev. C 4 (1971) 1977.

[10] Konecny, E., Specht, H. K. and Weber, J., Proceedings of the 3rd I.A.E.A. Symposium on the Physics and Chemistry of Fission, Vienne, Vol. II (1973) 3.
[11] Neuzil, E. F. and Fairhall, A. W., Phys. Rev. 126 (1963) 2705.

[12] Duffield, R. B., Schmitt, R. A. and Sharp, R. A., Rapport $\mathrm{P} / 678$, Proceedings of the 2 nd United Nations Conference on the Peaceful Uses of Atomic Energy, 15, Genève, 1958.

[13] Sugihara, T. T., Roesmer, J. and Meadows Jr., J. W., Phys. Rev. 121 (1961) 1179.

[14] Vandenbosch, R. and Huizenga, J. R., Phys. Rev. 127 (1962) 212

[15] Britt, H. C., Wegner, H. E. and GurSKy, J. C., Phys. Rev. 129 (1963) 2239.

[16] Croall, I. F. and Cuninghame, J. G., Nucl. Phys. A 125 (1969) 402.

[17] Unik, J. P. and Huizenga, J. R., Phys. Rev. 134B (1964) 90.

[18] Vandenbosch, R., Thomas, T. D., Vandenbosch, S. E., Glass, R. A. and Seabord, G. T., Phys. Rev. 111 (1958) 1358.

[19] Plasil, F., Burnett, D. S., Britt, H. C. and Thompson, S. G., Phys. Rev. 142 (1966) 696.

[20] Zhagarov, E. A., Kuks, I. M., Nemilov, Y. A., SelitSKII, Y. A. and FunsteIn, V. B., Nucl. Phys. A 213 (1973) 436. 